\title{
Lower cyclooxygenase-2 expression is associated with recurrence of solitary non-muscle invasive bladder carcinoma
}

Tomislav Tadin ${ }^{1,4^{*}}$, Kristian Krpina ${ }^{2}$, Sanja Štifter ${ }^{3}$, Emina Babarović ${ }^{3}$, Željko Fučkar ${ }^{2}$ and Nives Jonjić ${ }^{3}$

\begin{abstract}
Background: A new modality is necessary to prevent recurrence of superficial bladder cancer after complete transurethral resection because of the high recurrence rate even with current prophylaxis protocols.

Methods: In order to analyze the predictive value of cyclooxygenase-2 (COX-2) expression and tumor infiltrating lymphocytes (TILs) in recurrence of this disease tumor specimens from 127 patients with solitary papillary non-muscle invasive bladder cancer (NMIBC), 78 with recurrent disease and 49 without recurrence during follow up of minimum 5 years, were retrieved for tissue microarrays construction and immunohistochemical analysis. COX-2 expression was scored according to Allred's scoring protocol, while presence of TILs was categorized as absent (no) or present (yes) on whole tissue sections.
\end{abstract}

Results: COX-2 immunoreactivity was presented in 70 (71\%), weak in 16\% and strong in 55\% of cases, while 29 (29\%) tumors were negative. TILs were present in 64 (58\%) NMIBC, while 44 cases (41\%) did not reveal mononuclear infiltration in tumoral stroma. Statistical analysis demonstrated a higher proportion of patients with recurrence in the group with the COX-2 score 0 , and lower in the group with score 2 ( $p=0.0001, p=0.0101$, respectively). In addition, a higher proportion of recurrent patients in the group with no TILs, and lower proportion in the group with TILs were found ( $p=0.009, p=0.009$, respectively). Univariate and multivariate analysis revealed overexpression of COX-2 and presence of TILs as negative predictors.

Conclusion: Patients with lower COX-2 expression and absence of TILs in NMIBC need to be followed up more vigorously and probably selected for adjuvant therapy.

Virtual slide: The virtual slide(s) for this article can be found here: http://www.diagnosticpathology.diagnomx.eu/vs/ 1411318819790406

Keywords: Non-muscle invasive bladder cancer, Recurrence, Cyclooxygenase-2, Tumor infiltrating lymphocytes

\section{Background}

Urinary bladder cancer (UBC), on the average, includes $2 \%$ of all the malignant diseases with male-to-female ratio being about 4:1. The incidence of UBC increases with age [1]. The mortality from transitional cell carcinoma (TCC) of the urinary bladder increases significantly with the progression of superficial to invasive disease.

\footnotetext{
* Correspondence: medicina_ri@inet.hr

'Ultrasound Diagnostic Service, Health Centre Rijeka, Martina Kontuša 18, Rijeka 51000, Croatia

${ }^{4} T o m i s l a v$ Tadin, MD M.Sc., Ultrasound Diagnostic Service, Martina Kontuša 18, Rijeka 51000, Croatia

Full list of author information is available at the end of the article
}

Approximately $75-85 \%$ of patients present with a nonmuscle invasive bladder carcinoma (stages pTa, pT1, pTis). Despite the same category this is a very heterogeneous group of tumors with various biological outcomes. The main clinical feature of UBC is a high percentage of recurrence [2]. Carcinoma of the urinary bladder is the only malignant neoplasm for which immunotherapy is often included as part of standard care. Intravesical instillations of Bacille Calmette-Guerin (BCG) has been demonstrated to reduce the recurrence rate and the risk of progression to muscle-invasive disease in patients with carcinoma in situ (pTis), as well as non-muscle-invasive urothelial carcinomas [3,4]. BCG

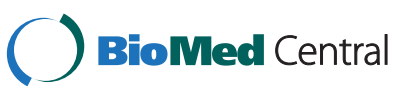


immunotherapy results in $70 \%$ to $75 \%$ and $50 \%$ to $60 \%$ complete response rates for carcinoma in situ and small residual tumors, respectively [5]. Unfortunately, a significant percentage of patients will fail initial BCG therapy. These patients would have much more benefit if they were oriented early to other therapeutic approaches. In addition, another $30 \%$ to $50 \%$ of BCG responders will develop recurrent tumors within 5 years $[6,7]$.

In fact, $70 \%$ of patients treated with transurethral resection (TUR) experience a relapse of the underlying disease and $15-25 \%$ of patients will progress over time to muscle-invasive cancer [2]. Although some prognostic variables have been shown to predict recurrence and can be used to identify patients who require adjuvant therapy after TUR, additional reliable markers for disease progression and recurrence are needed [8-10].

Standard prognostic factors like histological grading are limited in predicting possible recurrence of the disease. So, understanding of molecular processes that could reflect individual biological potential and clinical behavior is important. For that purpose, various biomarkers have been investigated since they have potential in decoding unique biological features in identifying patients with high risk of progression after the local treatment, as well as in more reliable prognosis and treatment of UBC [11]. In the present study the cyclooxygenase-2 (COX-2) was investigated. Beside prostaglandin $\mathrm{G} / \mathrm{H}$ synthases and $\mathrm{COX}-1$ it is one of the key enzymes in the synthesis of prostaglandins from arachidonic acid. COX-2 is not expressed in most tissue under normal conditions, but expression is rapidly induced by growth factors or agents that cause tissue irritation or inflammation [12]. COX-2 is expressed in many solid as well as hematological malignancies [13] where prostaglandins have been reported to increase proliferation, enhance angiogenesis, promote invasion, and inhibit apoptosis and differentiation, thus participating in carcinogenesis through various mechanisms $[13,14]$. There is evidence that COX-2 expression and activity is also important in the development of UBC $[15,16]$.

To determine if COX-2 overexpression could be causally related to recurrence of superficial UBC the present study was designed with pathologically homogenous group of solitary papillary non-muscle invasive bladder cancer (NMIBC) presented in patients according to development of the recurrent disease. More specifically, the aim was to assess and compare the COX-2 expression between the patients with and without recurrence of disease and, moreover, to analyze association between COX-2 expression and tumor infiltrating lymphocytes (TILs). We hypothesize that such investigation can be used to predict recurrence and help identify patients who require adjuvant treatment.

\section{Materials and methods Clinicopathological data}

The tumor specimens analyzed in this study were obtained from a total of 127 patients with solitary papillary NMIBC treated with initial transurethral resection (TUR), as a standard procedure at the Department of Urology, Rijeka University Hospital Center in Rijeka, between 1996 and 2006. None of the patients received adjuvant chemotherapy or immunotherapy or any other medical intervention after the initial TUR. All patients with multiple tumors and patients with a solid or flat aspect of the tumor were excluded from this study. The study was approved by the University of Rijeka Ethics Committee.

All the sections were reviewed to confirm the original diagnosis and were staged according to the 2002 American Joint Committee on Cancer guidelines [17] and graded according to the 2004 World Health Organization classification system [18] by an expert urologic pathologist. All the tumors were classified as papillary urothelial neoplasm of low malignant potential (PUNLMP) or low-grade papillary urothelial carcinoma (LGPUC). Clinicopathologic data were obtained from patient medical records and from the files kept at the Department of Pathology, Rijeka University School of Medicine. Based on disease recurrence, patients were divided in two groups: patients who developed recurrent disease during the first five post-operative years $(\mathrm{N}=78)$ and patients without recurrent disease during a follow-up of minimum 5 years $(\mathrm{N}=49)$. The follow-up of patients was subsequently scheduled at control cystoscopy every 3 months for the first two years after TUR, and after that biannually. Recurrence was defined as a written description of a recurrent tumor at any control cystoscopy 6 months after the operation, localized away from the primary tumor bed and the area of the initial resection. By this procedure protocol we wanted to exclude all possible residual tumor masses.

Hematoxylin and eosin stained tumor sections were used in order to evaluate the presence of inflammatory cells and to mark the areas with the most pronounced inflammatory infiltrate for construction of tissue microarrays. Positive inflammatory cells were semi quantitatively graded and presence of TILs was categorized as absent (no) or present (yes) as shown in Figure 1.

\section{Tissue microarray construction (TMA)}

Paraffin blocks were available for all 127 cases, and TMAs were constructed by using a manual tissue arrayer (Alphelys, Plaisir, France). Three tissue cores, each $1 \mathrm{~mm}$ in diameter, were placed into a recipient paraffin block. Normal liver tissue was used for orientation. Cores were spaced at intervals of $0.5 \mathrm{~mm}$ in the $\mathrm{x}$ - and $y$-axes. One section from each TMA block was stained with hematoxylin and eosin to confirm the presence of 


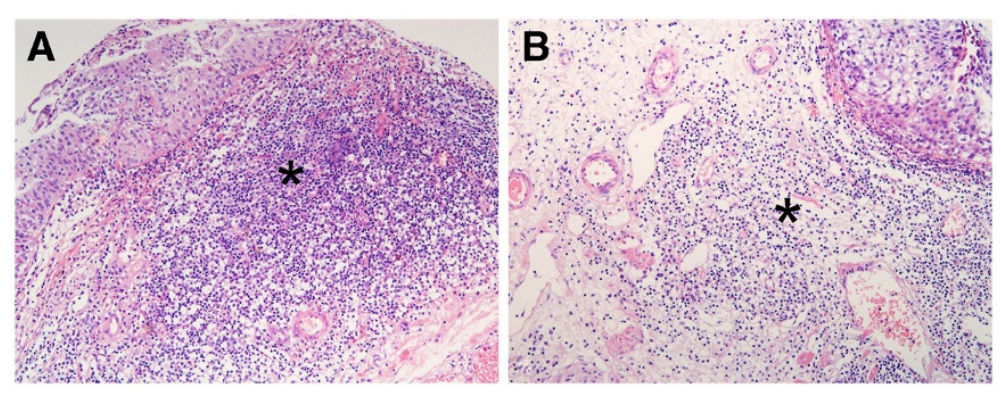

Figure 1 Urothelial tumors (hemalaun-eosin) with strong (A) and moderate (B) mononuclear inflammatory infiltrates in lamina propria $\left.{ }^{*}\right)$ predominantly composed of lymphocytes.

tumor tissue. Serial sections were cut from TMA blocks for immunohistochemical staining. Three to four $\mu \mathrm{m}$ thick sections were placed on adhesive glass slides (Capillary Gap Microscope Slides, $75 \mu \mathrm{m}$, Code S2024, DakoCytomation, Glostrup, Denmark), left to dry in oven at $55^{\circ} \mathrm{C}$ overnight, deparaffinized and rehydrated.

\section{Immunohistochemistry and scoring}

Tumor samples were processed for immunohistological analysis in a Dako Autostainer Plus (DakoCytomation Colorado Inc, Fort Collins, CO, USA) according to the manufacturer's protocol using Envision peroxidase procedure (ChemMate TM Envision HRP detection kit K5007, DakoCytomation, Glostrup, Denmark).

The sections were incubated with the primary antibodies, anti-COX-2 mouse monoclonal antibody (clone CX-294, code M3617, DAKO, Denmark), dilution range 1:100. The immune reaction was amplified using the appropriate secondary antibody and the Streptavidin-Biotin-Peroxidase HRP complex (code K5001, DAKO, Denmark). Sections were then developed using 3,3'-diaminobenzidine tetrahydrochloride chromogen (DAB, code K5001, DAKO, Denmark), under microscope control. The sections were counterstained with Mayer's Hematoxylin. Quality control performed by external and internal negative and positive controls was necessary to monitor the accuracy of tissue processing, staining procedures and reagents effectiveness. The primary antibody specificity sought to be assessed by their negative controls.

COX-2 immunohistochemical expression was quantified and scored by assessing a proportion of percentage as an intensity score according to Allred's scoring protocol [19]. The assigned proportion score represented the estimated proportion of positive-staining cells $(0$, none; $1,<1 / 100 ; 2,1 / 100$ to $1 / 10 ; 3,1 / 10$ to $1 / 3 ; 4,1 / 3$; to $2 / 3$; and $5,>2 / 3)$. The intensity score assigned represented the average intensity of positive cells $(0$, none; 1 , weak; 2 , intermediate; and 3 , strong). The proportion and intensity scores were combined to obtain a total score, which ranged from 0 to 8 . Total score $0-2$ was assessed as negative (0), score $3-5$ was assessed as weak positive (1) and 6-8 as strong positive (2). All slides were scored by investigator who was blinded to patient clinical information.

For the purpose of further statistical analysis, the mean values of immunohistochemical staining of all three tissue microarrays were used.

\section{Statistical analysis}

Statistical analysis was performed using MedCalc for Windows, version 12.3.0.0 (MedCalc Software, Mariakerke, Belgium). In the first part of the work the classical descriptive methods were used as well as the Chi-square test for the comparison of proportions. Mann-Whitney test was used to compare the medians. The method of logistic regression was used in order to compute the odds ratio for predictors of recurrence in univariate and multivariate manner. The AUC value was used to evaluate the quality of predictive model. The significance level in all tests was 0.05 .

\section{Results}

\section{Clinicopathological characteristics and} immunhistochemical findings in NMIBC

Clinicopathological characteristics of 127 patients with solitary, papillary NMIBC unrolled in the present study are summarized in Table 1. All clinical details along with adequate tumor samples, at the time of diagnosis and in follow up period, were available for all patients. None of the patients was lost to follow up. The median age at diagnosis for the patient cohort was 73 years (range 41-87), with a male to female ratio of 3.3:1, and a median follow up period of 37 months (range 6-155). Tumors divided according to their size in $\leq 3$ or $>3 \mathrm{~cm}$, according to their pathology in groups of papillary urothelial neoplasms of low malignant potential (PUNLMP) and low grade papillary urothelial carcinoma (LGPUC), and according to their stage in $\mathrm{Ta}$ and $\mathrm{T} 1$ were nearly equally distributed.

Immunohistochemical analysis revealed COX-2 expression predominantly granular localized to the cytoplasm of 
Table 1 Clinical features of patients with non-muscle invasive bladder carcinoma along with pathological and immunohistochemical characteristics of the resected tumors

\begin{tabular}{lc}
\hline Clinical, pathological and immunohistochemical features (N=127) \\
\hline Gender & Cases (\%) \\
Male & $94(74)$ \\
Female & $33(26)$ \\
Age (years, median, range) & $73(41-87)$ \\
Tumor size: & Cases (\%) \\
$>3 \mathrm{~cm}$ & $59(46)$ \\
$\leq 3 \mathrm{~cm}$ & $68(54)$ \\
Pathology: & Cases (\%) \\
PUNLMP & $54(47)$ \\
LGPUC & \\
Disease stage & $61(53)$ \\
Ta & Cases (\%) \\
T1 & $54(46 \%)$ \\
Median follow-up (months) & $64(54 \%)$ \\
COX-2 Allred score & 37 \\
0 & Cases (\%) \\
1 & $29(29)$ \\
2 & $16(16)$ \\
TILs* & $54(55)$ \\
No & Cases (\%) \\
Yes & $44(41)$ \\
\hline
\end{tabular}

Note: ${ }^{\dagger}$ PUNLMP indicates papillary urothelial neoplasm of low malignant potential; ${ }^{*}$ LGPUC, low-grade papillary urothelial carcinoma; ${ }^{*}$ TILs, tumorinfiltrating lymphocytes. tumor cells. Nuclear staining was not observed. In a few cases immunoreactivity was detected in endothelial cells within the tumor. Low intensity staining was also observed in smooth muscle tissue in some sections. There was noticeable heterogeneity in the intensity and percentage of COX-2 positive cells within the tumor tissues. Positive COX-2 immunoreactivity scored according to Allred was presented in $70(71 \%)$ while $29(29 \%)$ tumors were negative. Among positive tumors 16 (16\%) show weak and 54 (55\%) strong immunostaining. Representative cases of tumors with moderate and strong COX-2 staining are presented in Figure 2.

Tumor infiltrating lymphocytes as shown in Figure 1 were presented in majority $(64 ; 59 \%)$ of cases, while 44 cases $(41 \%)$ did not reveal mononuclear infiltration in tumoral stroma.

\section{COX-2 expression and TIL in correlation with recurrence of disease}

Main objectives of the present study were to examine possible association between COX-2 expression and TILs with regard to recurrence of disease, as well as their mutual relationship which revealed no significant association ( $\mathrm{p}=0.892)$.

Clinicopathological parameters included in the above mentioned analysis are presented in Table 2. As it can be seen among the examined parameters pathology and disease stages were different between recurrent and non recurrent disease. In particular, PUNLMP was more presented $(61 \%)$ among the non-recurrent disease in opposite to LGPUC that was more frequently present $(62 \%)$ in the recurrent group $(\mathrm{p}=0.0258, \mathrm{p}=0.0258$, respectively).

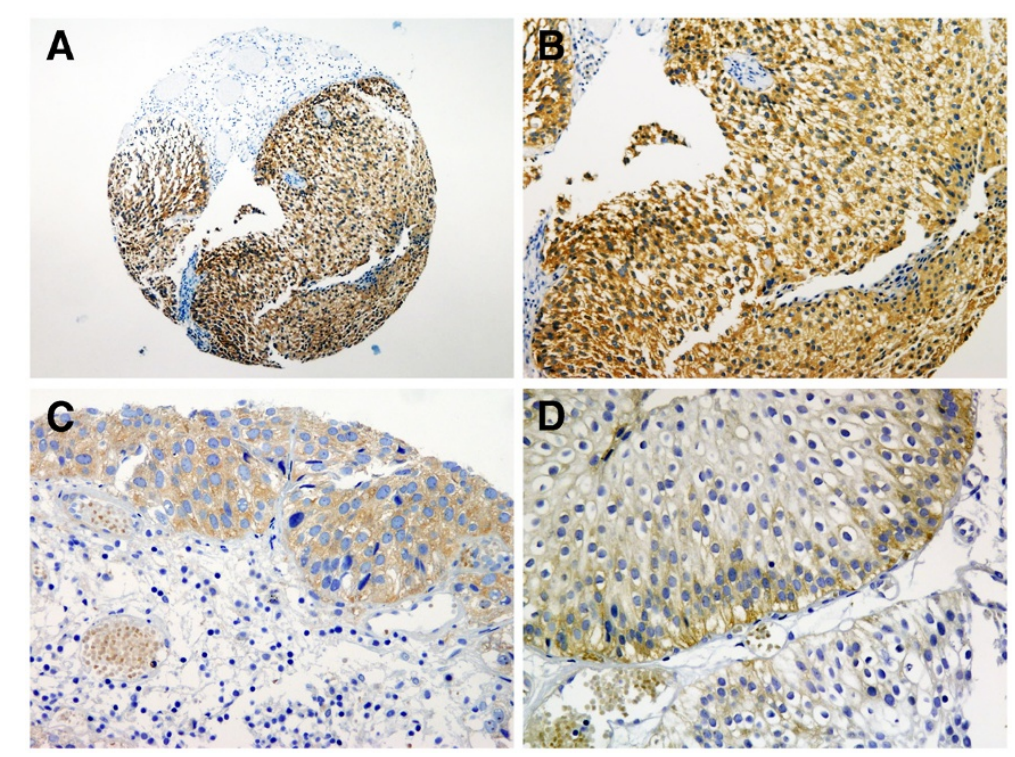

Figure 2 Immunohistochemical staining for COX-2 on tissue microarrays of urothelial tumors (A, magnification x20). On higher magnification representative cases demonstrate tumor cells with strong $(\mathbf{B})$, moderate $(\mathbf{C})$ and weak (D) staining (magnification $\times 100)$. 
Table 2 Clinicopathologic characteristics, COX-2 expression and TIL in comparison to recurrence of non-muscle invasive bladder carcinoma

\begin{tabular}{|c|c|c|c|}
\hline \multirow[b]{2}{*}{$\mathrm{N}=127$} & \multicolumn{2}{|c|}{ Recurrent disease } & \multirow[b]{2}{*}{ P-value } \\
\hline & Yes $(n=78)$ & No $(n=49)$ & \\
\hline Gender & Cases (\%) & Cases (\%) & \\
\hline Male & $58(74)$ & $36(73)$ & 0.8834 \\
\hline Female & $20(26)$ & $13(27)$ & \\
\hline Age (years, median, range) & $71(41-87)$ & $67(54-78)$ & \\
\hline Tumor size: & Cases (\%) & Cases (\%) & \\
\hline$>3 \mathrm{~cm}$ & $40(51)$ & $19(39)$ & 0.1104 \\
\hline$\leq 3 \mathrm{~cm}$ & $38(49)$ & $30(61)$ & \\
\hline Pathology: & Cases (\%) & Cases (\%) & \\
\hline PUNLMP $^{\dagger}$ & $26(38)$ & $28(61)$ & 0.0258 \\
\hline LGPUC $^{\ddagger}$ & $43(62)$ & $18(39)$ & \\
\hline Disease stage & Cases (\%) & Cases (\%) & \\
\hline $\mathrm{Ta}$ & $26(37)$ & $28(61)$ & 0,0182 \\
\hline T1 & $46(63)$ & $18(39)$ & \\
\hline Median follow-up (months) & 13 & 75 & \\
\hline COX-2 score & Cases (\%) & Cases (\%) & \\
\hline 0 & $27(45)$ & $2(5)$ & 0.0001 \\
\hline 1 & $7(12)$ & $9(23)$ & 0.2196 \\
\hline 2 & $26(43)$ & $28(72)$ & 0.0101 \\
\hline TILs* & Cases (\%) & Cases (\%) & \\
\hline No & $32(53)$ & $12(26)$ & 0.009 \\
\hline Yes & $29(47)$ & $35(74)$ & \\
\hline
\end{tabular}

Note: ${ }^{\top}$ PUNLMP indicates papillary urothelial neoplasm of low malignant potential; ${ }^{*}$ LGPUC, low-grade papillary urothelial carcinoma; * tumorinfiltrating lymphocytes.

According to disease stages there were more patients with recurrence in stage T1 (63\%), while those patients without recurrent disease were in stage $\mathrm{Ta}(61 \%)$ $(\mathrm{p}=0.018)$.

Moreover, statistical analysis demonstrated a difference in tumors COX-2 expression between the analyzed groups, e.g. between recurrent and non-recurrent patients. Possibly, due to small sample size in the group with the score 1 no significant difference was found between recurrent and non-recurrent patients $(\mathrm{p}=0.2196)$. In the group with the score 0 and the group with the score 2 there was a significant difference in the proportion of patients with and without recurrence $(\mathrm{p}=0.0001$, $\mathrm{p}=0.0101$, respectively). From these results we can expect that lower score is predictor of recurrence, since there is higher proportion of recurrent patients with score 0 and lower proportion of recurrent patients with score 2 .

A significant difference between the recurrent and nonrecurrent patients in both groups, with absent and present TILs was also found ( $\mathrm{p}=0.009, \mathrm{p}=0.009$, respectively).
More precisely, higher proportion of recurrent patients in the group with no TILs and lower proportion in the group with TILs were found. Therefore, we can expect the absence of TILs to be the predictor of recurrence.

\section{Univariate and multivariate analysis of prediction of recurrence}

Univariate and multivariate analysis of the influence of COX-2 expression, TILs, tumor size and disease stage on prediction of recurrence (PR) are shown in Table 3.

The odds ratio for each predictor was computed using the method of logistic regression. As we can see the tumor size is not the significant predictor in univariate, as well as is in multivariate manner. Disease stage is in both analysis significant and positive predictor, which means that the odds for a recurrence in cases with stage 1 are 2.39 times higher than in cases with stage 0 . In both manners the TILs and COX-2 are negative predictors. The positive change in score for COX-2 (score higher for 1) produces 0.36 times lower risk, or that the odd for not getting a recurrence is $1 / 0.36=2.77$ times higher. The TILs is also significant negative predictor of recurrence with OR 0.31 in univariate manner and 0.23 in multivariate analysis. The AUC value as a classification parameter which gives us the quality of predictive model was used. In our case we have the AUC 0.875 which means that our model has very high discriminating power.

\section{Discussion}

There is large evidence implicating COX-2 activity in the development of UBC. Yet, the results obtained in the present study demonstrated that low COX-2 instead of high COX-2 score could predict the recurrence of NMIBC. These results open many questions related to the methodology used in various studies, as well as the complexity in understanding the role of different biological markers in tumorigenesis.

In the present study we used TMA instead of whole tissue section for immunohistochemical analysis. Gudjonsson et al. in their study analyzed COX-2 among different markers on TMA and they found that proteins assessed had no predictive value for recurrences of Ta bladder cancer (BC) [20]. The concerns have been raised by the authors regarding the methodology and generalization of results obtained with TMA in immunohistochemical analysis. Even so we believe that conclusion was made despite the facts that relatively small number of patients $(\mathrm{N}=52)$ was analyzed. TMA construction was done with at least three $0.6 \mathrm{~mm}$ punch cores and specimens were heterogeneous, including single and multifocal tumors. In order to minimize these problems the present study was conducted on relatively larger number of the patients $(\mathrm{N}=127)$, with only single tumor at the time of diagnosis, and with 
Table 3 Predictors of recurrence: univariate and multivariate analyses

\begin{tabular}{|c|c|c|c|c|c|c|c|}
\hline \multirow[t]{2}{*}{ Predictor } & \multicolumn{4}{|c|}{ Univariate } & \multicolumn{3}{|c|}{ Multivariate } \\
\hline & OR & $95 \% \mathrm{Cl}$ & P-value & AUC & OR & $95 \% \mathrm{Cl}$ & P-value \\
\hline Tumor size & 1.69 & {$[0.82 ; 3.49]$} & 0.1541 & 0.565 & 1.71 & {$[0.62 ; 4.69]$} & 0.3007 \\
\hline Disease stage & 2.39 & {$[1.15 ; 4.98]$} & 0.0194 & 0.607 & 3.29 & {$[1.18 ; 9.19]$} & 0.0228 \\
\hline TILs & 0.60 & {$[0.40 ; 0.89]$} & 0.0122 & 0.651 & 0.49 & {$[0.29 ; 0.84]$} & 0.0092 \\
\hline COX-2 & 0.36 & {$[0.20 ; 0.63]$} & 0.0004 & 0.691 & 0.27 & {$[0.14 ; 0.53]$} & 0.0002 \\
\hline
\end{tabular}

Note: $O R=$ Odds ratio; $C l=$ Confidence interval; $A U C=$ Area under the curve; $T I L s=$ tumor-infiltrating lymphocytes.

specimens where at least three $1 \mathrm{~mm}$ punch could be selected for TMA construction. Nevertheless, the obtained results indicate that different methodology and also diverse scoring system for protein expression could be the reason for different results and conclusion made in different papers.

Previous studies have been conducted mostly on heterogeneous groups of patients with superficial and invasive UBC. One of the first papers in which the expression of COX-1 and COX-2 were analyzed in human invasive TCC of the urinary bladder samples was by Mohammed and coworkers [21]. COX-2 was not expressed in normal urinary bladder samples but was detected in invasive TCC, noninvasive TCC samples, and in cases of carcinoma in situ. Authors concluded that COX-2 may play a role in $\mathrm{BC}$ and support further study of COX-2 inhibitors as potential antitumor agents in human BC. After this study several other ones showed significant increase of COX-2 expression with advancing tumor grade and $\mathrm{T}$ stages of the disease [22,23], and not only with disease progression but also with $\mathrm{BC}$ specific survival [24].

Opposite to the role of COX-2 in tumor progression there are also studies where COX-2 expression was not associated with primary tumor stage, lymph node status, histological grading, overall and disease-free survival [25]. Wulfing et al. also did not find COX-2 expression associated with TNM staging, histological grading, overall or disease free survival, but a significant relation to the histological subtype (transitional vs. squamous cell carcinoma) was present [26]. Subgroup of chemotherapy patients demonstrated a significant correlation of strong COX-2 expression with worse overall survival time. The authors concluded that further experimental and clinical studies were needed to elucidate if COX-2 inhibition can serve as an additive therapy to chemotherapy of $\mathrm{BC}$.

There are also several studies analyzing COX-2 expression only in NMIBC. In the CIS group, COX-2 expression was significantly associated with disease recurrence and progression, but not with $\mathrm{BC}$ related survival (as in our analysis, data not shown), while in the stage T1 the TCC COX-2 expression was not associated with clinical or pathological parameters or clinical outcome [27]. Kim and coworkers in their study selected only T1G3 TCC who had undergone complete TUR [28]. In that case COX-2 expression was statistically significant in predicting both recurrence and disease progression, while patients' age, shape and multiplicity of tumors were not significantly predictive. Thus patients, according to authors' conclusion, with COX-2 positive superficial BC may need to be followed up more vigorously. In the paper of Okajima et al. with only 5 and 6 samples of superficial BC cases with and without recurrence, respectively, after TUR, more COX-2 protein samples in the cases with recurrence than in cases without recurrence were found [29]. Even though the number of cases examined is small, as the authors stated, this result supports their hypothesis that COX-2 contributes to superficial $\mathrm{BC}$ recurrence, thus selective COX-2 inhibitors can be a candidate chemo preventive agents for reoccurrence.

Our results differ from the above mentioned because loss of COX-2 was a predictive marker of tumor recurrence. We believe this can be explained by very complex, even different role of this enzyme during tumorigenesis. There is no doubt that COX-2 expression is sequentially up-regulated from normal to chronic cystitis and to malignant changes [30]. Also there are evidence that COX2 is involved in angiogenesis in $\mathrm{BC}$, as described in the paper where COX-2 promoted vessel proliferation in the tumor zone of pTa/pT1 NMIBC [31]. Moreover, COX-2 is probably up-regulated during tumor progression, as mentioned above, but we believe its role in initial stage is very complex. We hypothesize that, in the initial tumor stage, COX-2 is probably associated with host immunological/inflammatory response and, as such, it could be a marker of sufficient anti-tumor response while loss of COX-2 expression may be indication for the selection of patients for additional immunotherapy. This supposition is also supported by our finding that absence of TILs became the predictor of recurrence. In our study we could not find the association between COX-2 and TILs although there is study by Himly and coworkers in which the association between COX-2 expression and $\mathrm{T}$ lymphocyte subsets, $\mathrm{CD} 4+$ and $\mathrm{CD} 8+$, was confirmed [23]. However, our previous work confirmed the proportion of CD4+ cells and Granzyme B+ 
TILs being significantly higher in non-recurrent group of patients [32].

\section{Conclusion}

Although further studies are necessary to fully elucidate the mechanism involved, the present study showed that higher COX-2 expression and present TILs resulted as a negative predictor of recurrence in NMIBC. On the other hand, patients with lower value of both analyzed parameters should be probably selected for adjuvant therapy.

\section{Competing interests}

The authors declare that they have no competing interests.

\section{Authors' contributions}

TT participated in study design, conceived the study and participated in coordination. KK added clinical data. SŠ actively performed part of immunohistochemical analysis. EB conceived the study and participated in coordination and performed statistical analysis. ŽF participated in study design and coordination. NJ advised and led the whole group as coordinator. All authors read and approved the final manuscript.

\section{Acknowledgements}

We thank our technicians Ozren Štanfel and Tihomila Bušić for their excellent technical assistance. This work was supported by the Ministry of Science, Education and Sports of the Republic of Croatia (grant number 062-06200950082).

\section{Author details}

'Ultrasound Diagnostic Service, Health Centre Rijeka, Martina Kontuša 18, Rijeka 51000, Croatia. ²Department of Urology, University Hospital Centre Rijeka, Tome Strižića 3, Rijeka 51000, Croatia. ${ }^{3}$ Department of Pathology, Faculty of Medicine, University of Rijeka, B. Branchetta 20, Rijeka 51000, Croatia. ${ }^{4}$ Tomislav Tadin, MD M.Sc., Ultrasound Diagnostic Service, Martina Kontuša 18, Rijeka 51000, Croatia.

Received: 19 August 2012 Accepted: 30 October 2012

Published: 5 November 2012

\section{References}

1. Siegel R, Ward E, Brawley O, Jemal A: Cancer statistics, 2011: The impact of eliminating socioeconomic and racial disparities on premature cancer deaths. CA Cancer J Clin 2011, 61(4):212-236.

2. Amling CL: Diagnosis and management of superficial bladder cancer. Curr Probl Cancer 2001, 25(4):219-278.

3. Witjes JA, Hendricksen K: Intravesical pharmacotherapy for non-muscleinvasive bladder cancer: a critical analysis of currently available drugs, treatment schedules, and long-term results. Eur Urol 2008, 53(1):45-52.

4. Brandau S, Suttmann H: Thirty years of BCG immunotherapy for nonmuscle invasive bladder cancer: a success story with room for improvement. Biomed Pharmacother 2007, 61(6):299-305.

5. Böhle A, Bock PR: Intravesical bacillus Calmette-Guerin versus mitomycin $C$ in superficial bladder cancer: formal meta-analysis of comparative studies on tumour progression. Urology 2004, 63(4):682-687.

6. Sylvester RJ, van der Meijden AP, Oosterlinck W, Witjes JA, Bouffioux C, Denis $L$, et al: Predicting recurrence and progression in individual patients with stage Ta T1 bladder cancer using EORTC risk tables: a combined analysis of 2596 patients from seven EORTC trials. Eur Urol 2006, 49:466-477.

7. Babjuk M, Oosterlinck W, Sylvester R, Kaasinen E, Böhle A, Palou J, et al: EAU guidelines on non-muscle-invasive urothelial carcinoma of the bladder, the 2011 update. European Association of Urology (EAU). Eur Urol 2011, 59(6):997-1008.

8. Fernandez-Gomez J, Madero R, Solsona E, Unda M, Martinez-Piñeiro L, Gonzalez $M$, et al: Predicting nonmuscle invasive bladder cancer recurrence and progression in patients treated with bacillus CalmetteGuerin: the CUETO scoring model. J Urol 2009, 182(5):2195-2203.
9. Böhle A, Brandau S: Immune mechanisms in bacillus Calmette-Guerin immunotherapy for superficial bladder cancer. J Urol 2003, 170(3):964-969.

10. Rodríguez-Faba O, Palou J: Predictive factors for recurrence progression and cancer specific survival in high-risk bladder cancer. Curr Opin Urol 2012, 22(5):415-420.

11. Matsushita K, Cha EK, Mtsumt K, Baba S, Chromecki TF, Fajkovic H, Sun M, Karakiewitc PI, Scherr DS, Shariat SF: Immunohistochemical biomarkers for bladder cancer prognosis. Int J Urol 2011, 18(9):616-629.

12. Kam PC, See AU: Cyclo-oxygenase isoenzymes: physiological and pharmacological role. Anaesthesia 2000, 55:442-449.

13. Barisik NO, Bozkurt S, Gumus M, Kaygusuz I, Karadayi N, Bas E, Bayik M, Tecimer T: Expression and prognostic significance of cox-2 and p-53 in Hodgkin lymphomas: a retrospective study. Diagn Pathol 2010, 5:19.

14. Marks F, Fustenberg G: Cancer chemoprevention through interruption of multistage carcinogenesis. The lessons learnt by comparing mouse skin carcinogenesis and human large bowel cancer. Eur J Cancer 2000, 36:314-329

15. Shirahama T: Cycloxygenase-2 expression is up-regulated in transitional cell carcinoma and its preneoplastic lesins in the human urinary bladder. Clin Cancer Res 2000, 6:2424-2430.

16. Klein RD, Van Pelt CS, Sabichi AL, dela Cerda J, Fischer SM, Furstenberger G, Muller-Decker K: Transitional cell hyperplasia and carcinomas in urinary bladders of transgenic mice with keratin 5 promoter-driven cyclooxygenase-2 overexpression. Cancer Res 2005, 65(5):1808-1813.

17. Greene FL, Page DL, Fleming ID, Fritz A, Balch CM: AJCC Cancer Staging Manual. 6th edition. Philadelphia: Lippincott-Raven; 2002:335-337.

18. Eble JN, Sauter G, Epstein JI, Sesterhenn IA: World Health Organization Classification of Tumours: Pathology and Genetics of Tumours of the Urinary System and Male Genital Organs Lyon. France: IARC Press; 2004.

19. Kulkarni S, Rader JS, Zhang F, Liapis H, Koki AT, Masferrer JL, Subbaramaiah K, Dannenberg AJ: Cyclooxygenase- 2 is overexpressed in human cervical cancer. Clin Cancer Res 2001, 7(2):429-434

20. Gudjonssn S, Bendahl PO, Chebil G, Hogllund M, Lindgren D, Lundberg LM, Lovgren K, Ferno M, Manssn W, Liedberg F: Can tissue microarray-based analysis of protein expression predict recurrence of stage Ta bladder cancer? Scand J Urol Nephrol 2011, 45(4):270-277.

21. Mohammed SI, Knapp DW, Bostwick DG, Foster RS, Khan KNM, Masferrer UL, Woerner BM, Snyder PW, Koki AT: Expression of cyclooxygenase -2 (COX-2) in human invasive transitional cell carcinoma (TCC) of the urinary bladder. Cancer Res 1999, 59:5647-5650.

22. Wadhwa P, Gswami AK, Joshi K, Sharma SK: Cycloxygenase-2 expression increases with the stage and grade in transitional cell carcinoma of the urinary bladder. Int Urol Nephrol 2005, 37:47-53.

23. Himly M, Campbell R, Bartlett JMS, McNicol AM, Underwood MA, McMillan DC: The relationship between the systemic inflammatory response, tumour proliferative activity, T-lymphocytic infiltration and COX-2 expression and survival in patients with transitional cell carcinoma of the urinary bladder. British J Cancer 2006, 95:1234-1238.

24. Shariat SF, Matsumot K, Kim J, Ayala GE, Zhou JH, Jian W, Benedict WF, Lerner SP: Correlation of cycloxyfgenase-2 expression with molecular markers, pathlogical features and clinical outcome of transitional cell carcinoma of the bladder. J Urol 2003, 170:985-989.

25. Eltze E, Wulfing C, Von Struensee D, Piechota H, Buerger H, Hertle L: COX-2 and Her2/neu co-expressi in invasive bladder cancer. Int J Oncol 2005, 26:1525-1531.

26. Wulfing $C$, Eltze E, Von Struensee D, Wulfing P, Bode ME, Bettendorf O, Piechota $\mathrm{H}$, Herle L: Cyclooxygenase-2 expression in bladder cancer: tumor-biological and clinical implications. Aktuelle Urlo/ 2004, 35:331-338

27. Shariat SF, Kim JH, Ayala GE, Kho K, Wheeler TM, Lerner SP: Cycloxygenase2 is highly expressed in carcinoma in situ and $T 1$ transitional cell carcinoma of the bladder. J Urol 2003, 169:938-942.

28. Kim SI, Kwon SM, Kim Y, Hng SI: Association of cyclooxygenase-2 expression with prognosis of stage T1 grade 3 bladder cancer. Urology 2002, 60(5):816-821.

29. Okajima E, Uemura H, Ohnishi S, Tanaka M, Ohta M, Tani M, Fujimoto K Ozono S, Okajima E, JHirao Y: Expression of cyclooxygenase-2 in primary superficial bladder cancer tissue may predict risk of its recurrence after complete transurethral resection. Aktuelle Urol 2003, 34(4):256-258.

30. Moussa M, Omran Z, Nosseir M, Lotfy A, Swellam T: Cyclooxygenase-2 expression on urothelial and inflammatory cells of cystoscopic biopsies 
and urine cytology as possible predictive marker for bladder carcinoma. APMIS 2009, 117(1):45-52.

31. Fridrich MG, Tma MI, Petri S, Huland H: Cyclooxygenase-2 promotes angiogenesis in $\mathrm{pTa} / \mathrm{T} 1$ urothelial bladder carcinoma but does not predict recurrence. BJU Int 2003, 92(4):389-392.

32. Krpina K, Babarović E, Đorđević G, Fučkar Ž, Jonjić N: Recurrence of solitary non-muscle invasive bladder cancer is associated with mucosaassociated CD8 positive lymphocytes. CMJ, in press.

doi:10.1186/1746-1596-7-152

Cite this article as: Tadin et al:: Lower cyclooxygenase-2 expression is associated with recurrence of solitary non-muscle invasive bladder carcinoma. Diagnostic Pathology 2012 7:152

\section{Submit your next manuscript to BioMed Central and take full advantage of:}

- Convenient online submission

- Thorough peer review

- No space constraints or color figure charges

- Immediate publication on acceptance

- Inclusion in PubMed, CAS, Scopus and Google Scholar

- Research which is freely available for redistribution 